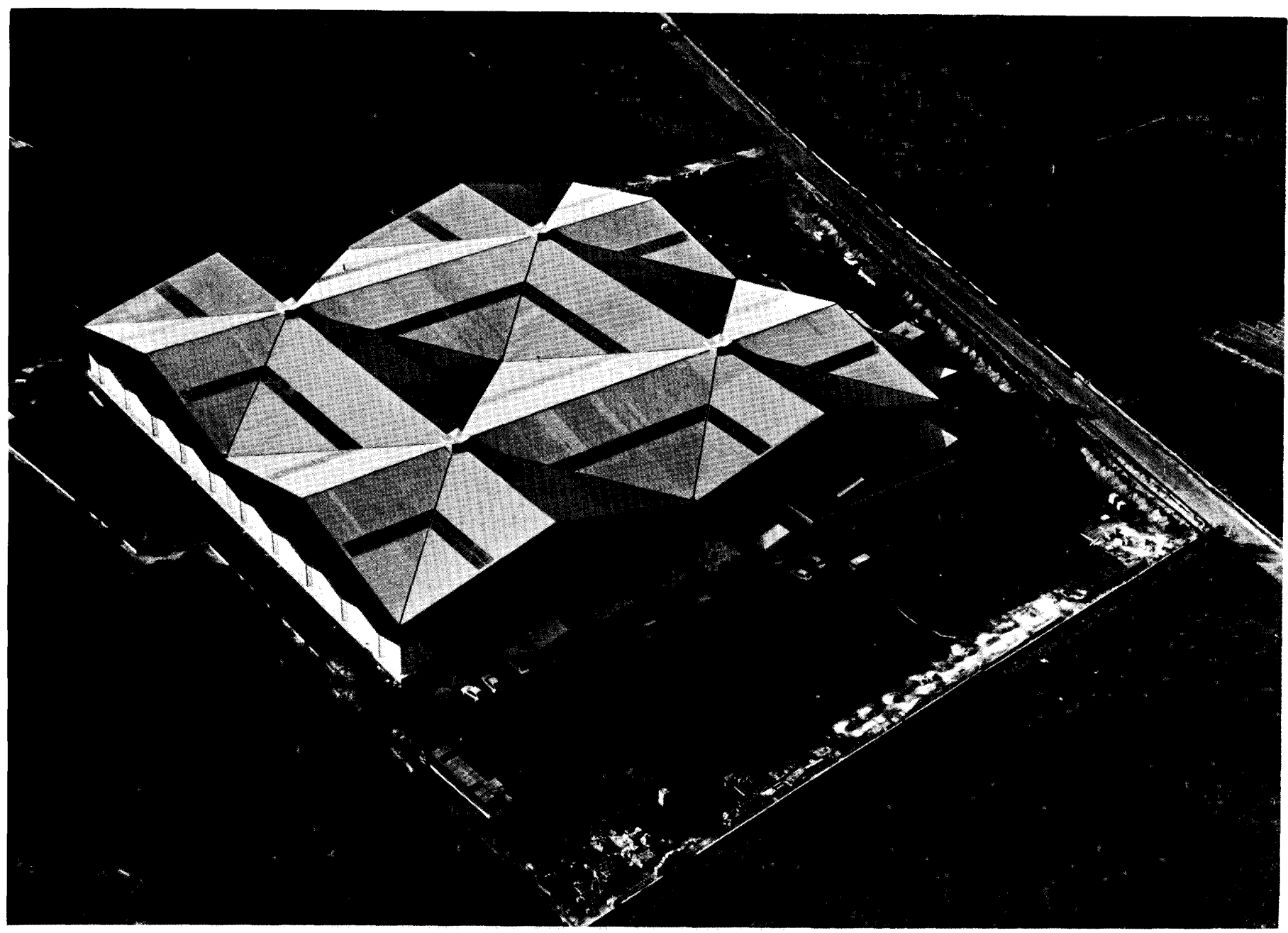

\title{
estructura metálica de la Central Hortofrutícola en Beniarjó
}

\section{(España)}

841-23

sinopsis

Se presenta la solución original de esta nave, de planta cuadrada, que ha me recido el premio Sercola 730 , de $100 \mathrm{~m}$ do lado y 7,30 m do altura Gil, resuelta a base de un estructura metalica espacial formada por planos una lámina plegada.
unclinas

Se cumplen todas las pre misas establecidas: $\theta \mathrm{Co}$ nomía y competitividad con las convencionales que dejan los mismos os pacios libres material do cubierta tradiciont cubior tradicional, ovacuacion de aguas corrocta, aspocto extemo a interno agradables $y$, en re sumen, armonia entre la funcionalidad técnica $y$ estética, lo que constituye un logro excepcional o menos no demasiado fre cuente.

\section{ANTECEDENTES}

Don Vicente y don Pascual Giner Navarro encargaron a los Ingenieros Agrónomos don Carlos M. Ferrer y Ferrer y don José S. Martínez Navarro la redacción del proyecto de una Central Hortofrutícola en el término municipal de Beniarjó (Valencia), cuya estructura fue dimensionada por don Carlos Ferrer y Ferrer y don Carlos A. Martínez Lasheras.

El programa establecido por la propiedad consistia fundamentalmente en una edificación principal de $10.000 \mathrm{~m}^{2}$ destinados a manipulación de agrios para una exportación de 30.000 t/año y cámaras frigoríficas de unas 2.000 t de capacidad de conservación a $0^{\circ} \mathrm{C}$, con edificios auxiliares para oficinas, comedores y demás dependencias de personal asi como zonas de acceso, carga y descarga lo más amplias posibles. Para ello la propiedad aportaba un solar de $23.000 \mathrm{~m}^{2}$ de forma sensiblemente rectangular.

\section{JUSTIFICACION Y DESCRIPCION DE LA SOLUCION ADOPTADA}

El "Lay-out" fijado después de un minucioso estudio comparativo de diversas alternativas fue el siguiente: 


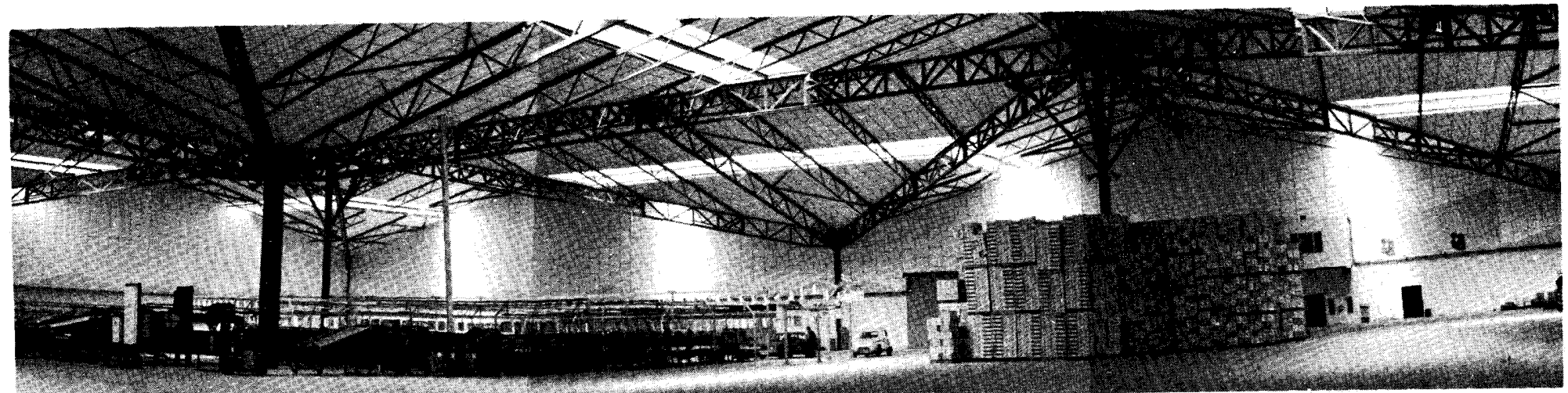

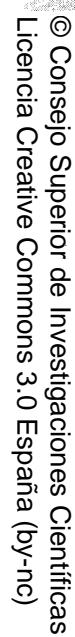
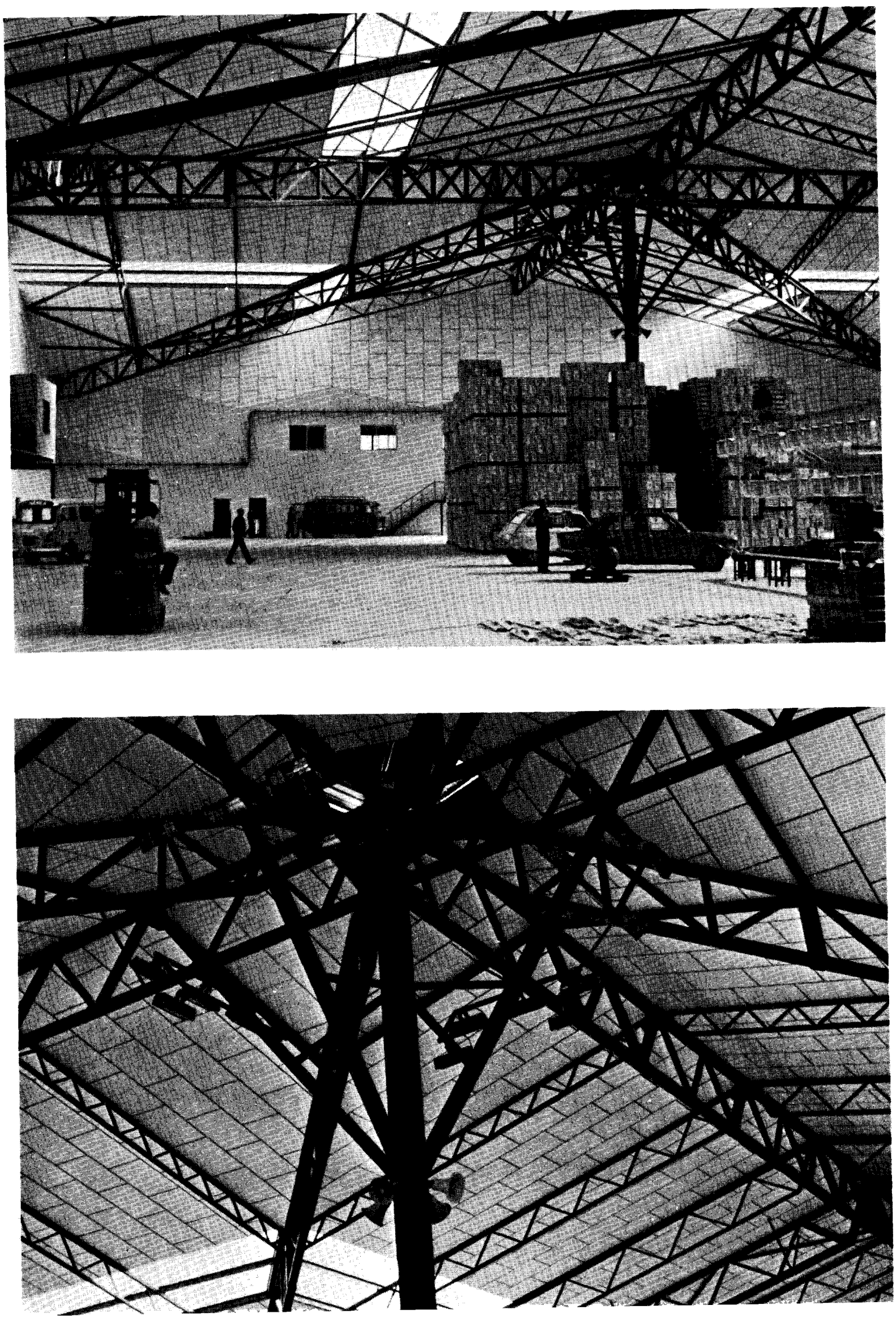
a) Edificio de planta cuadrada de $100 \mathrm{~m}$ de lado y 7,30 de altura útil y elevado $1 \mathrm{~m}$ aproximadamente sobre el parque de carga y descarga, con el condicionamiento de limitar al máximo el número de soportes para facilitar la colocación de la maquinaria, el movimiento y almacenamiento interiores.

b) Edificio de oficinas con conexión con el interior de la nave de manipulación y situado en la fachada principal norte junto al muelle de expedición.

c) Edificio de servicios al personal, en la fachada este y contiguo al edificio de oficinas junto al cual se encuentra el muelle de recepción.

d) El bloque de cámaras frigorificas queda emplazado en el cuadrante suroeste del edificio principal y tiene unas dimensiones en planta de $35 \mathrm{~m} \times 45 \mathrm{~m}$.
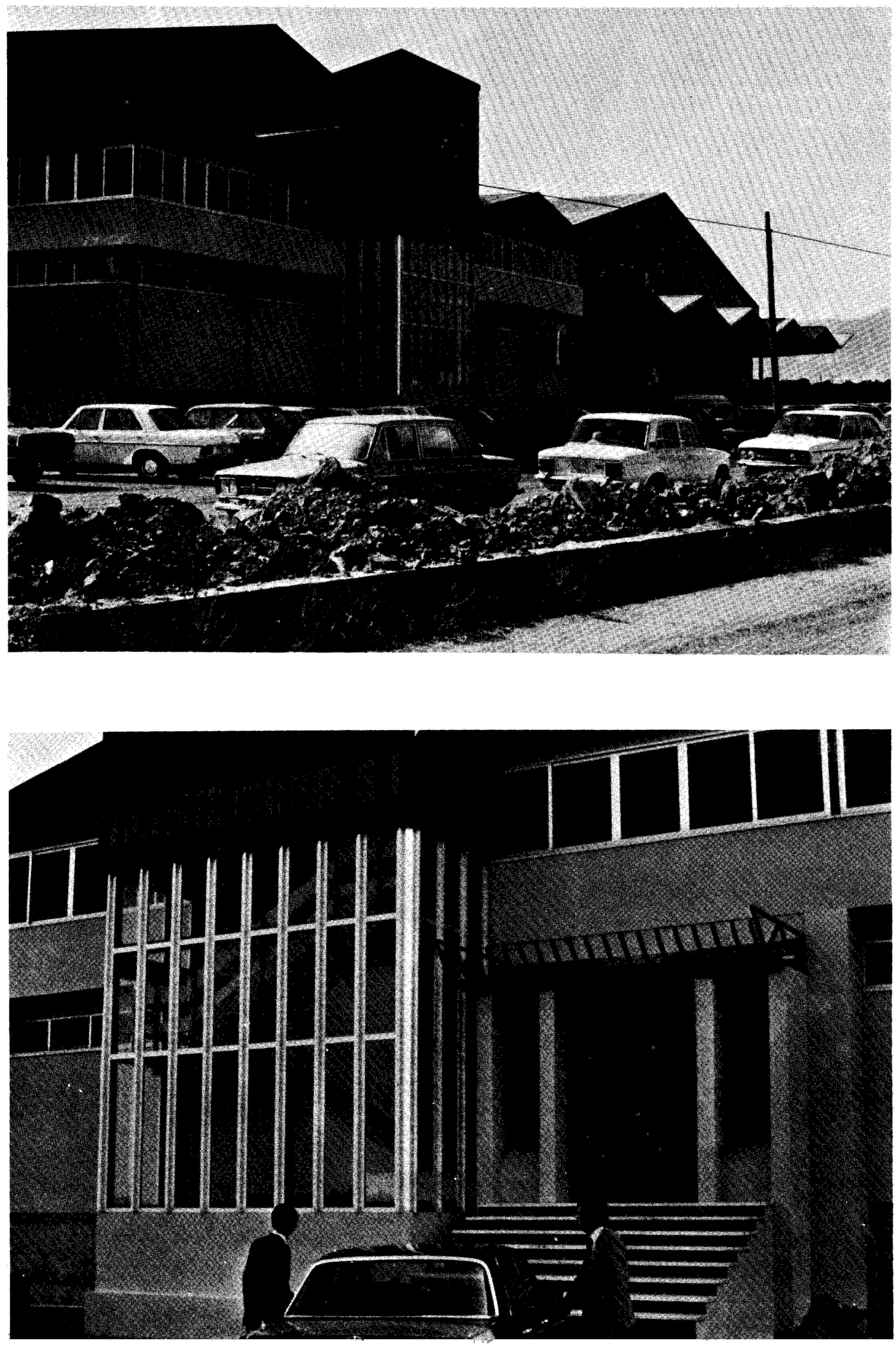


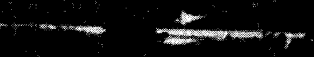

Ante este planteamiento, una solución estructural clara se ofrecia a los proyectistas: una malla espacial de superficie superior cuasi plana, apoyada en las cuatro fachadas con soportes interiores. Evidentemente, es la solución más económica en cuanto a peso de acero estructural pero no así cuando se consideran los costos de ejecución en taller y montaje. También es necesario considerar el costo del material de cobertura para una solución de tan pequeña pendiente. Está claro que este punto se hubiera soslayado con una malla espacial con gran pendiente, pero hubiera llevado a alturas en cumbrera excesivamente grandes encerrando un gran volumen inútil.

Se desecharon asimismo soluciones en cúpula porque una planta circular o elíptica del edificio no convenía a los condicionamientos principales de proyecto.

Tambièn se estudiaron otras soluciones laminares de hormigón armado, pero su costo para grandes luces se elevaba extraordinariamente a la vez que el plazo de ejecución se dilataba respecto del de cualquier solución en estructura metálica.

Por otro lado, se desecharon las soluciones de naves paralelas con cubiertas a dos aguas por demasiado convencional. No se pensó una estructura en diente de sierra, que si bien es la solución idónea en cuanto a iluminación, es cara, tanto de construcción como de mantenimiento.

Una solución aparecía como muy interesante: la de una estructura espacial formada por planos inclinados, es decir, una lámina plegada. Muchas posibilidades se estudiaron que sería interminable describirlas; sólo nos detendremos en explicar la solución adoptada.
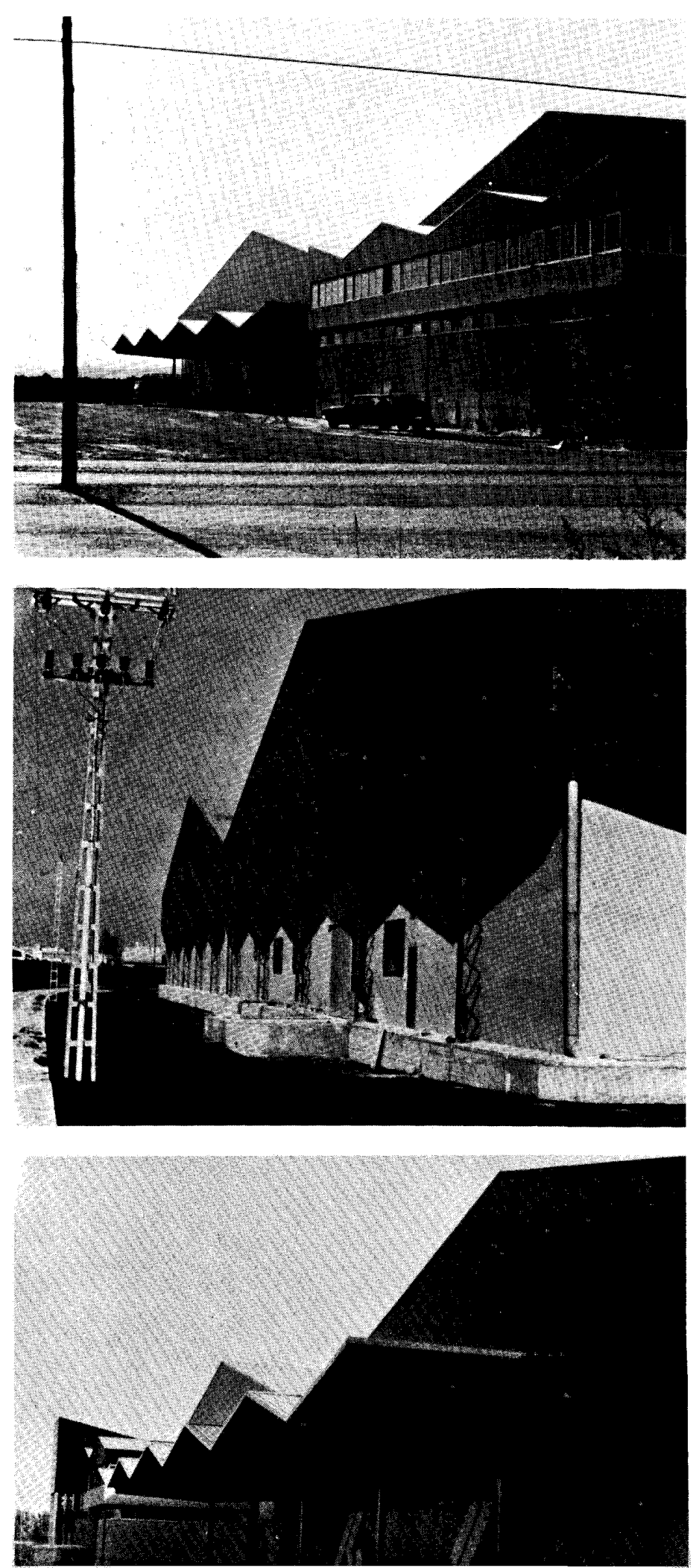


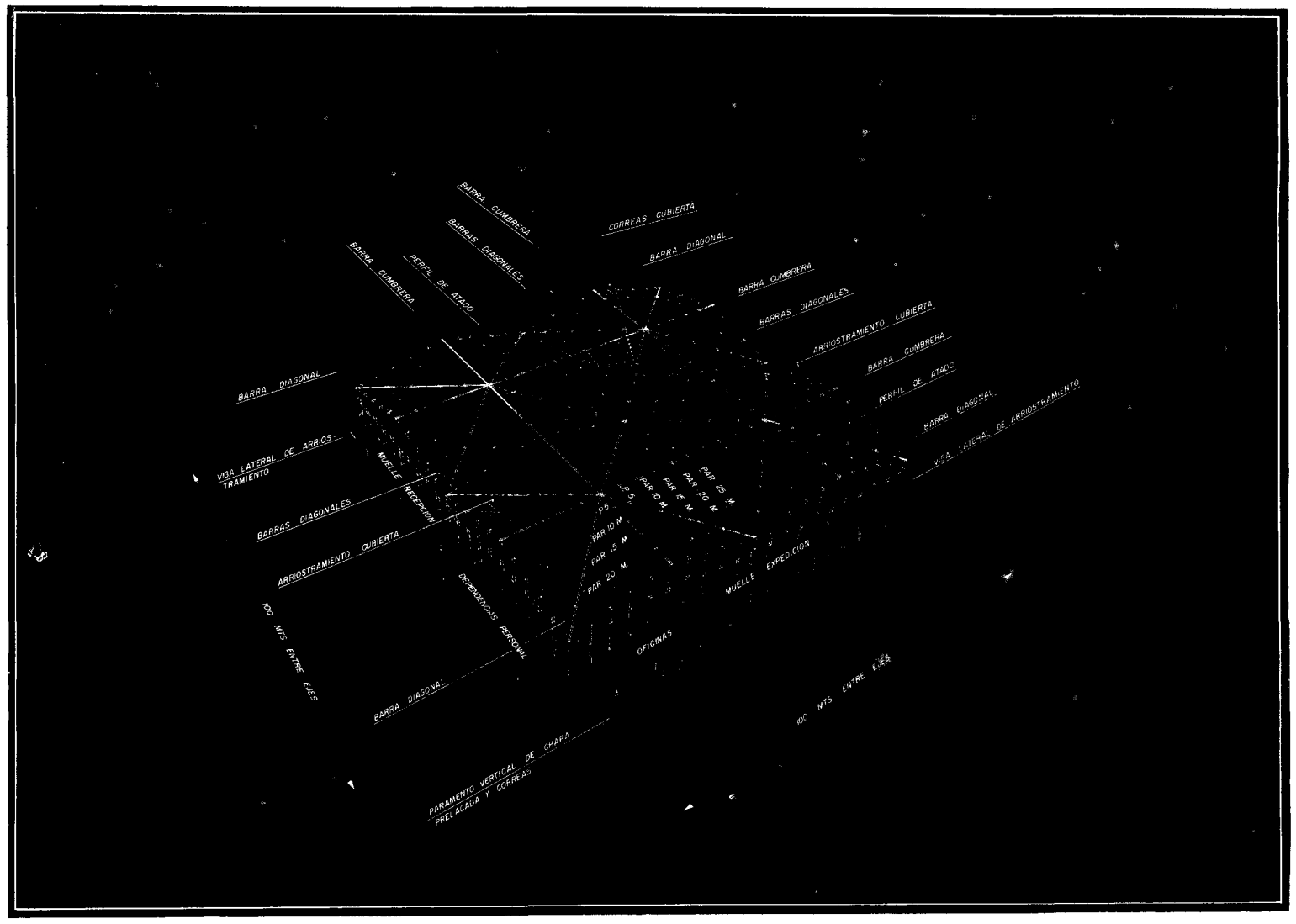

La cubierta tiene cuatro limatesas horizontales en líneas paralelas a las fachadas y situadas a $25 \mathrm{~m}$ de ellas. Desde los puntos de intersección de estas líneas salen 16 limahoyas en direcciones paralelas a las diagonales que van a parar a 9 puntos bajos, uno situado en el centro geométrico de la nave y los restantes en las cuatro esquinas y en los puntos medios de las lineas de fachadas. Estas líneas delimitan los planos de cubierta como queda ilustrado en el plano correspondiente.

La estructura portante principal está, pues, compuesta de las barras de cumbrera, las barras diagonales, los pares así como de los perfiles de atado de la fachada. Está apoyada en soportes situados en las fachadas y en sólo cinco soportes interiores: uno en el centro de la nave y el resto en las verticales de las intersecciones de las limatesas.

Las correas llevan las cargas verticales a los pares cuya misión es doble: por un lado rigidizan los grandes planos de cubierta y de ahí su rigidez a flexión y por otro impide la flexión de las barras de cumbrera y de las diagonales; es decir, transforman las cargas verticales en acciones tangenciales según los planos de cubierta.

Las diagonales trabajan fundamentalmente a esfuerzos axiles y la relativa rigidez proporcionada a estos elementos es para limitar al máximo los puntales provisionales de montaje. Dada la configuración geométrica de la lámina los mayores esfuerzos que soportan estos elementos los proporcionan los pares principales de $25 \mathrm{~m}$ y las vigas de atado de fachada.

Las barras de cumbrera soportan pequeños esfuerzos cuando actúan sólo las cargas verticales simétricas. Se han dimensionado con una mayor rigidez de la estrictamente necesaria, por las mismas consideraciones de montaje antes expuestas para las diagonales, asi como para proporcionar una suficiente estabilidad de la lámina cuando estuviere sometida a las acciones horizontales de viento y a las muy poco probables cargas asimétricas de nieve.

Todos estos elementos están dimensionados con perfiles de tubo que proporcionan una mayor economía en material y en tiempo de ejecución. 

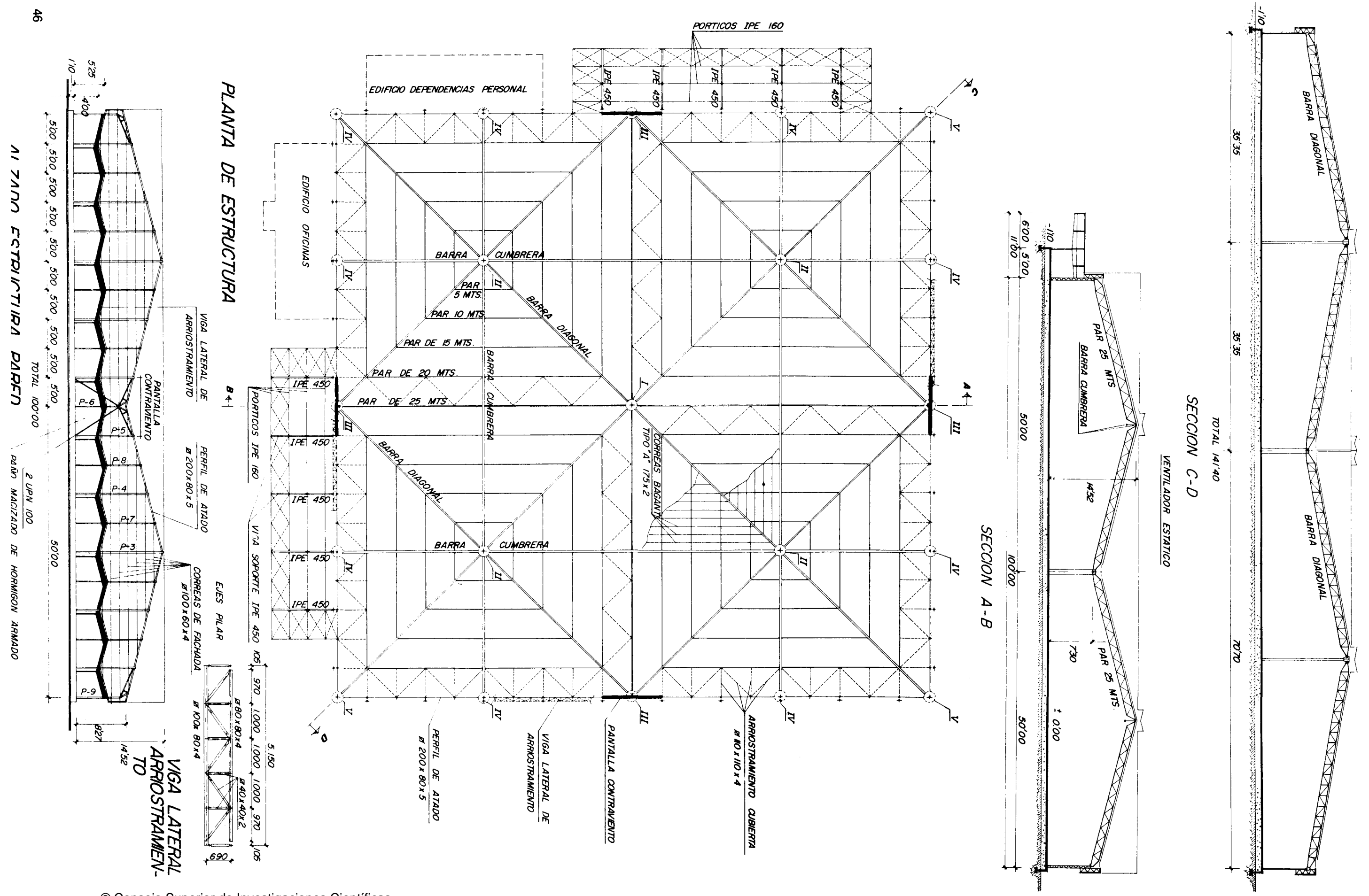

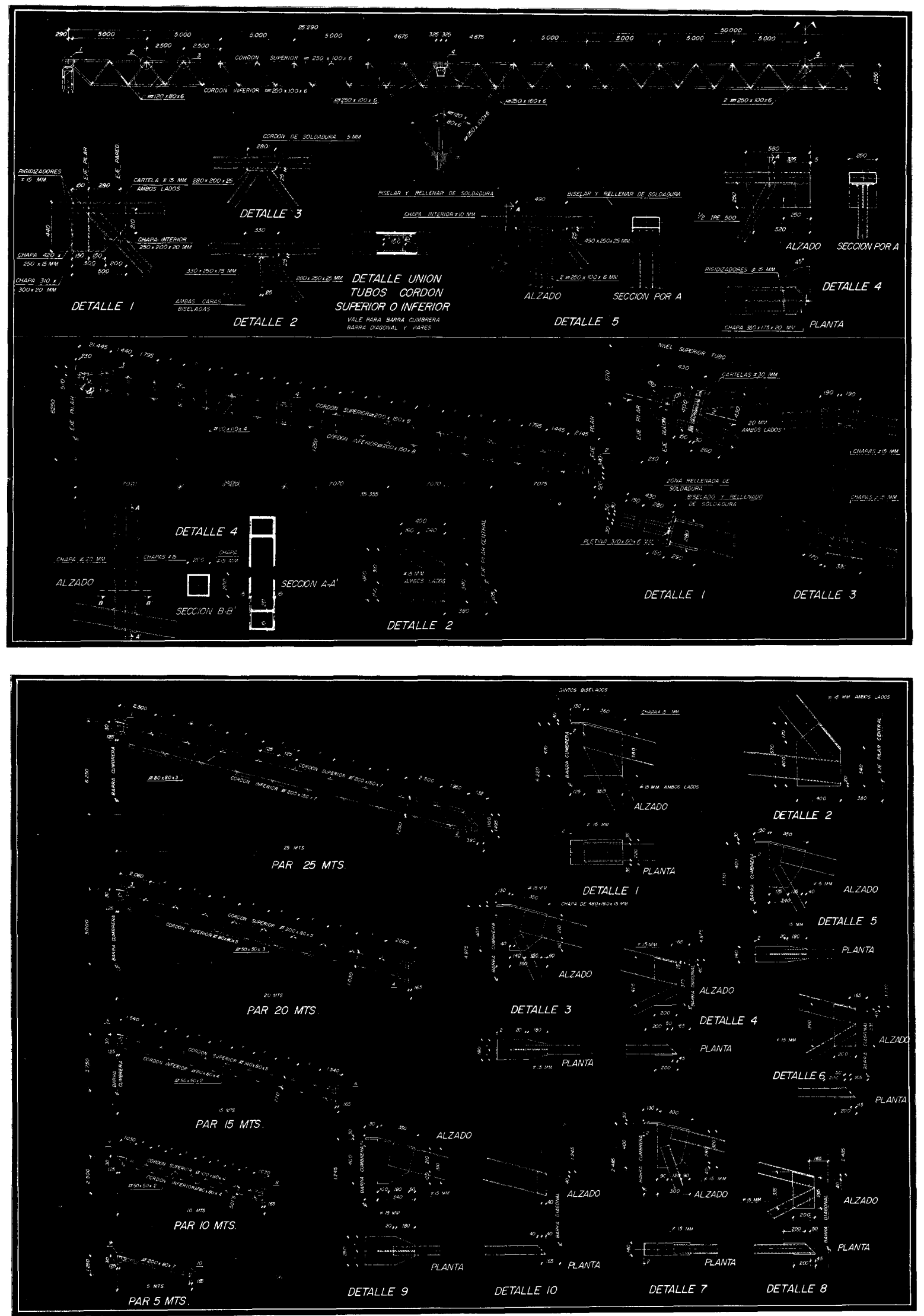

El soporte central tiene forma tubular $y$, concéntrico con él, se halla la bajante de aguas fluviales que recoge una cuenca de $2.500 \mathrm{~m}^{2}$. Los dos tubos se han rigidizado entre si, mediante un relleno de hormigón que colabora en parte en el trabajo del soporte. Los otros cuatro soportes interiores se han diseñado mediante la unión de cuatro perfiles que recogen perfectamente los empotramientos de las barras de cumbrera. Dada su gran esbeltez se han arriostrado a la cubierta mediante jabalcones. 

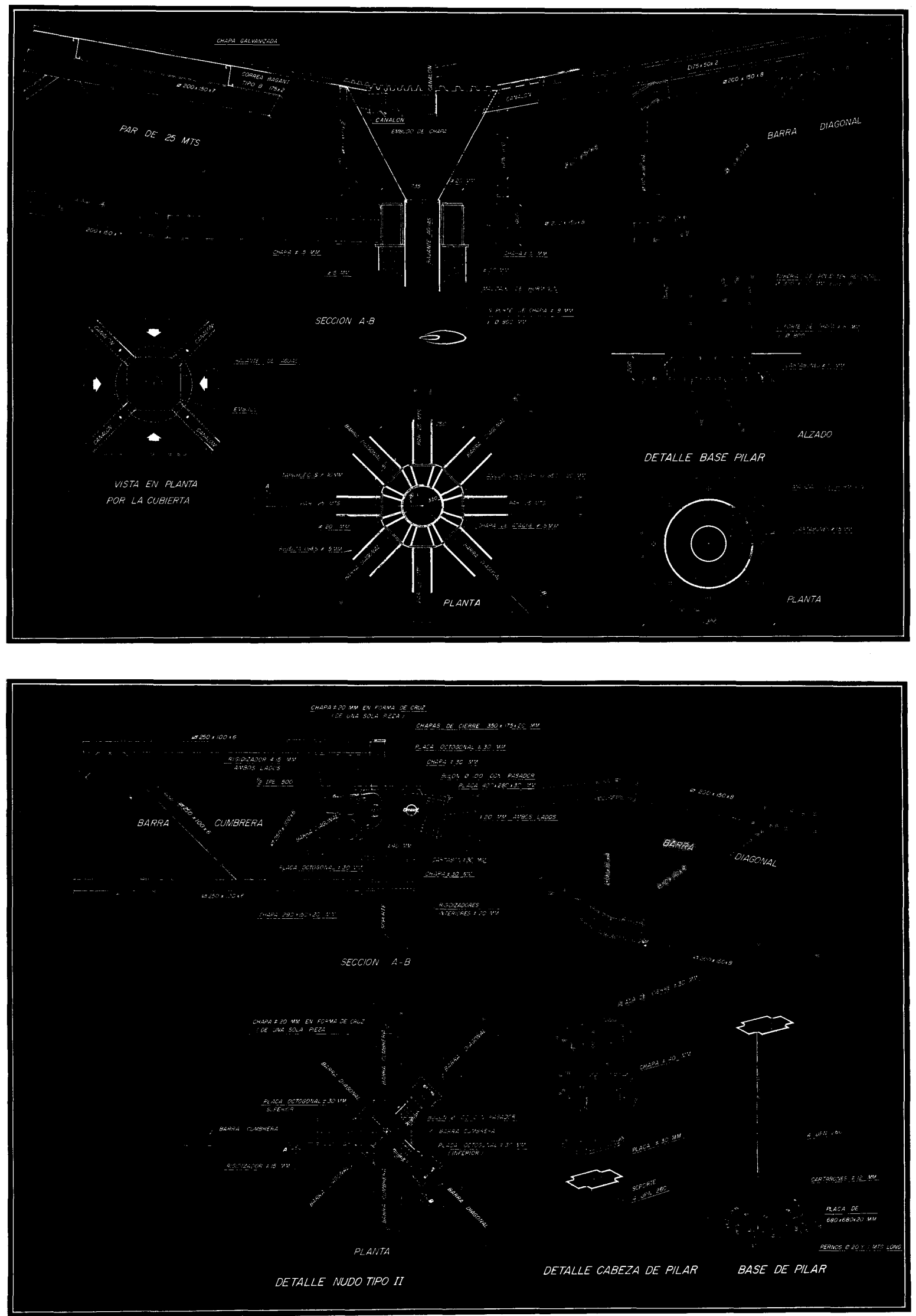

Los soportes perimetrales que recogen las cargas de los nudos de la lámina y las reacciones de la barra perimetral, y que a su vez rigidizan la fachada respecto a las acciones del viento se han proyectado con tubo siendo alternativamente uno simple y otro compuesto de barras trianguladas entre si, como se desprende de la observación de planos y fotografías. 

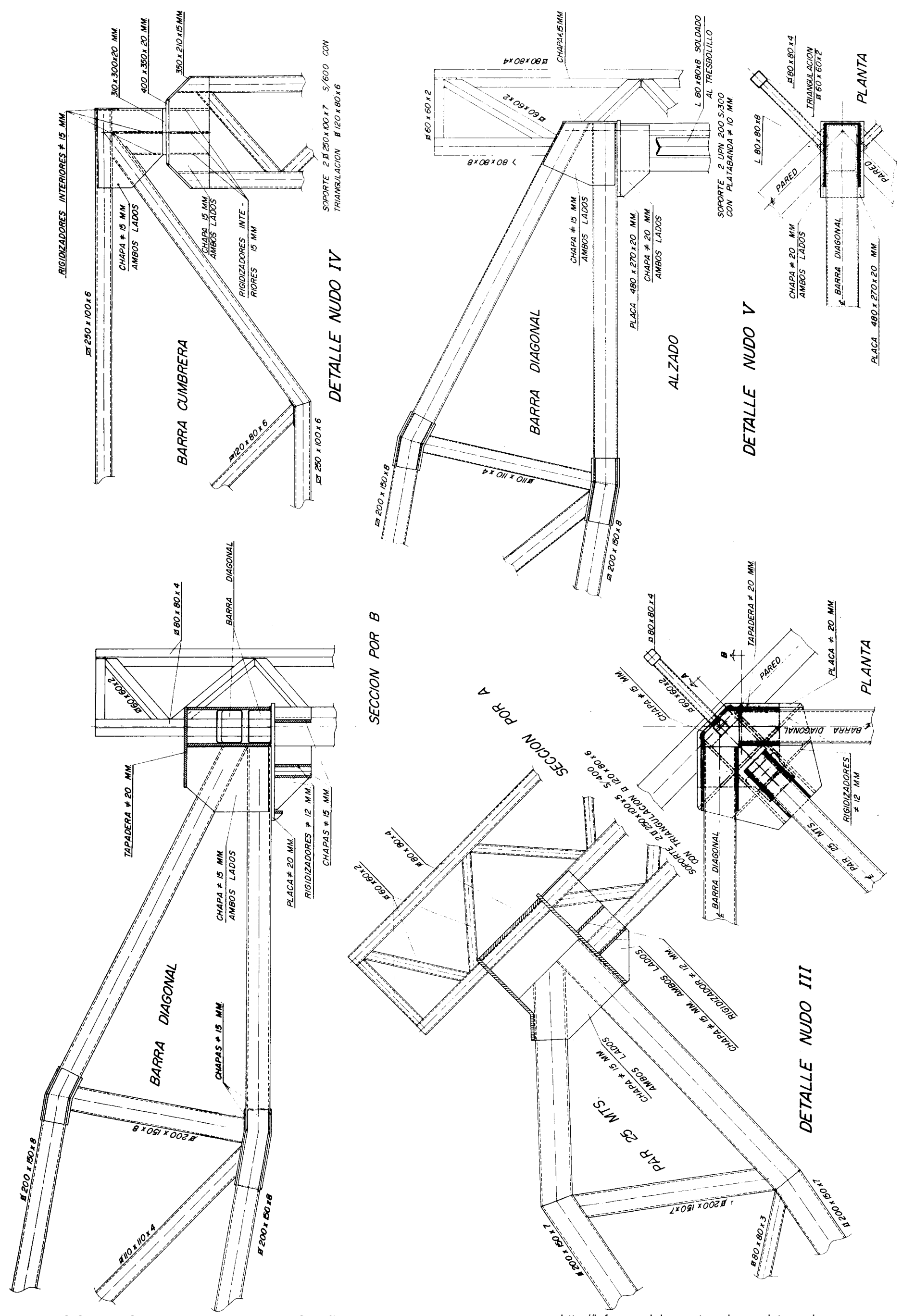

$\infty$
0
8
2
0
0
4
5 

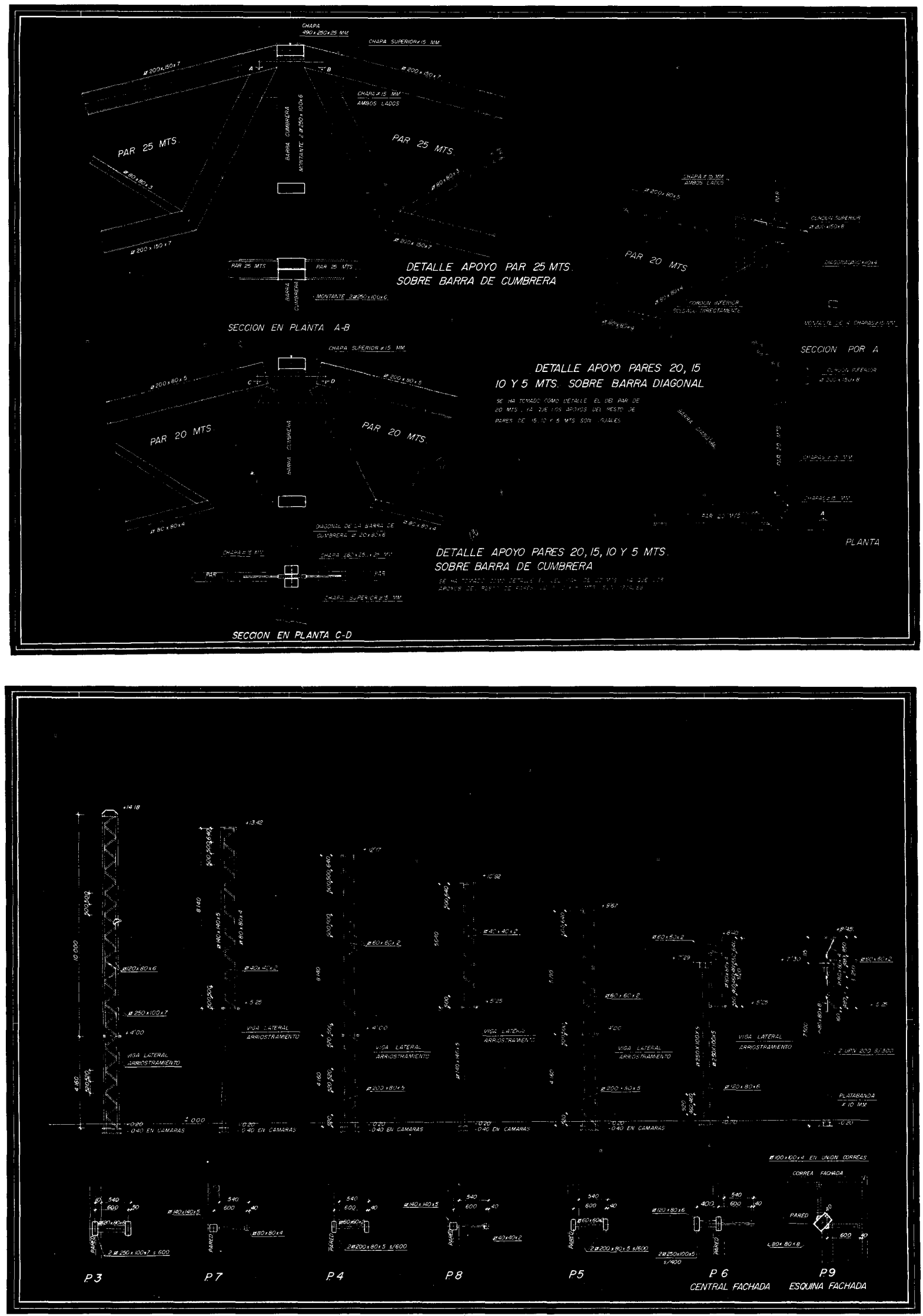

Los edificios anejos tienen una estructura convencional con formas armónicas con la del edificio principal. Asimismo forman una unidad con éste las marquesinas de gran vuelo situadas en las zonas de recepción de materia prima y de expedición. 


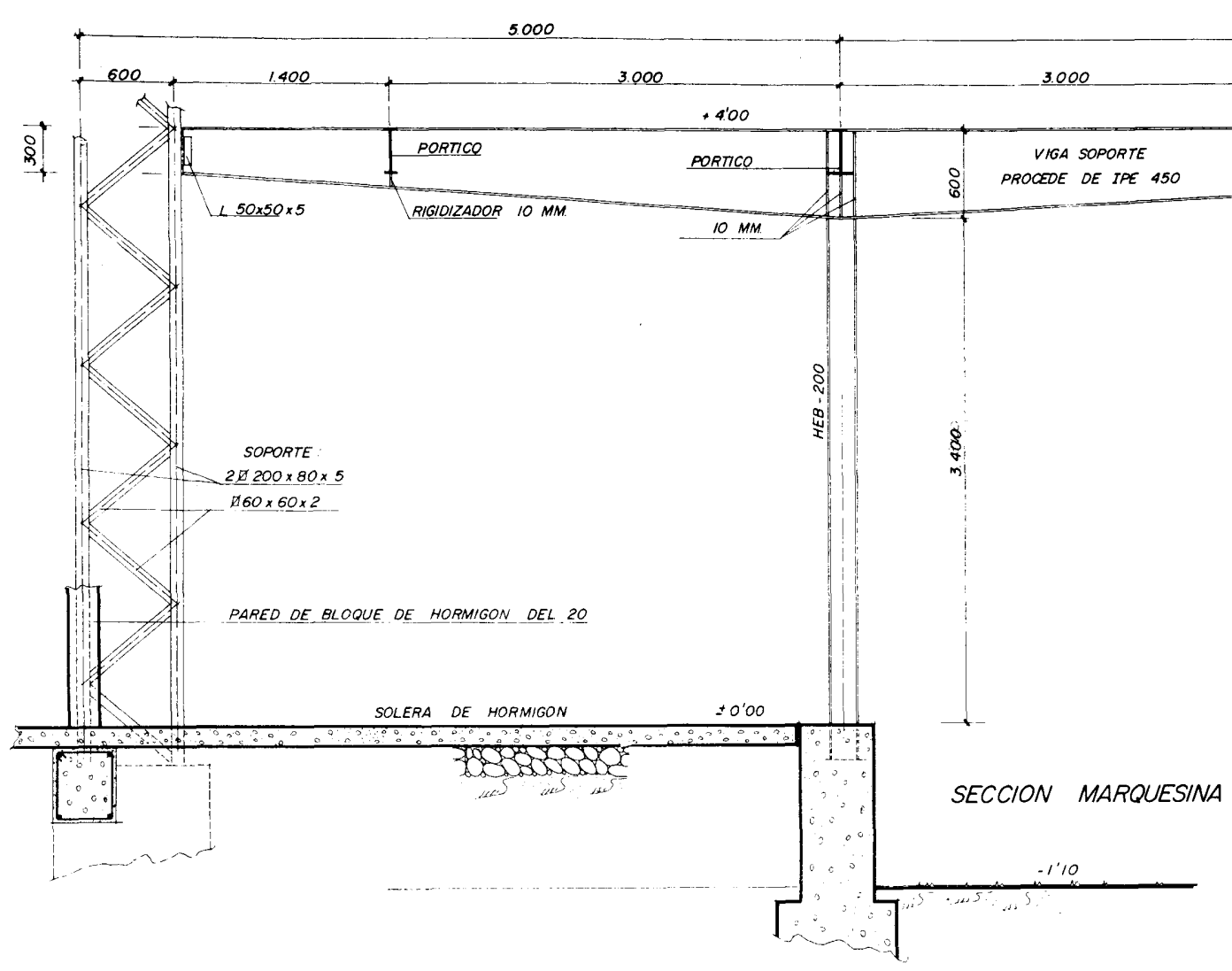

DETALLES MARQUESINA

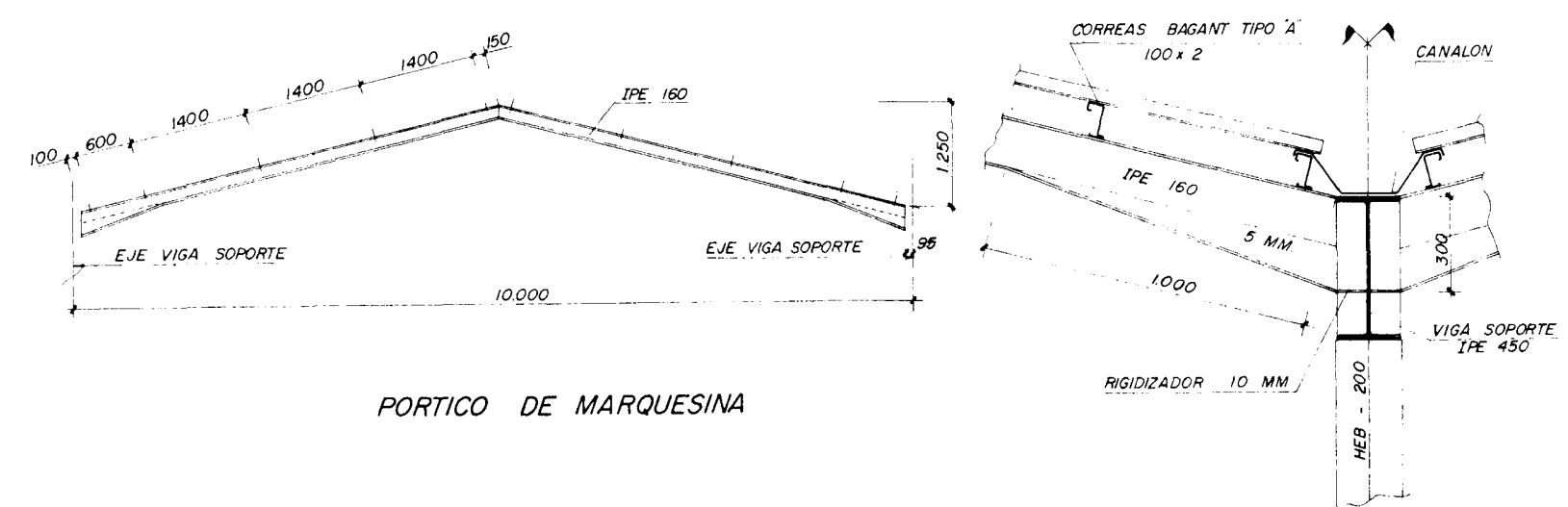


barras de cumbrera. El montaje se realizó de esta manera con una gran sencillez y sólo tuvo especial importancia el montaje de las barras de cumbrera que se unieron en el suelo y se elevaron en piezas de $50 \mathrm{~m}$.

El descimbrado de la estructura se fue haciendo ordenadamente, primero cortando por el pie los puntales de las diagonales y posteriormente los puntales de las barras de cumbrera. Aqui se observó el buen trabajo de la lámina puesto que las mayores deformaciones observadas fueron de unos $2 \mathrm{~cm}$ cuando las piezas tomadas aisladamente habrian flectado más de $10 \mathrm{~cm}$. En su construcción ya se habia previsto la correspondiente contraflecha.

\section{CONCLUSIONES}

La estructura, que fue premiada en el concurso convocado por la Convención Europea de la Construcción Metálica en su fase nacional, a través de SERCOMETAL, es muy original.

Se han cumplido mediante una feliz concepción los siguientes objetivos:

- Solución económica y competitiva con cualquier otra convencional que dejara tan amplios espacios libres.

- Material de cubierta convencional sin impermeabilización y por tanto económico.

- Evacuación de aguas de cubierta muy sencilla y sin riesgo de humedades interiores.

- Estructura integrada en la arquitectura.

- Aspecto estético muy agradable.
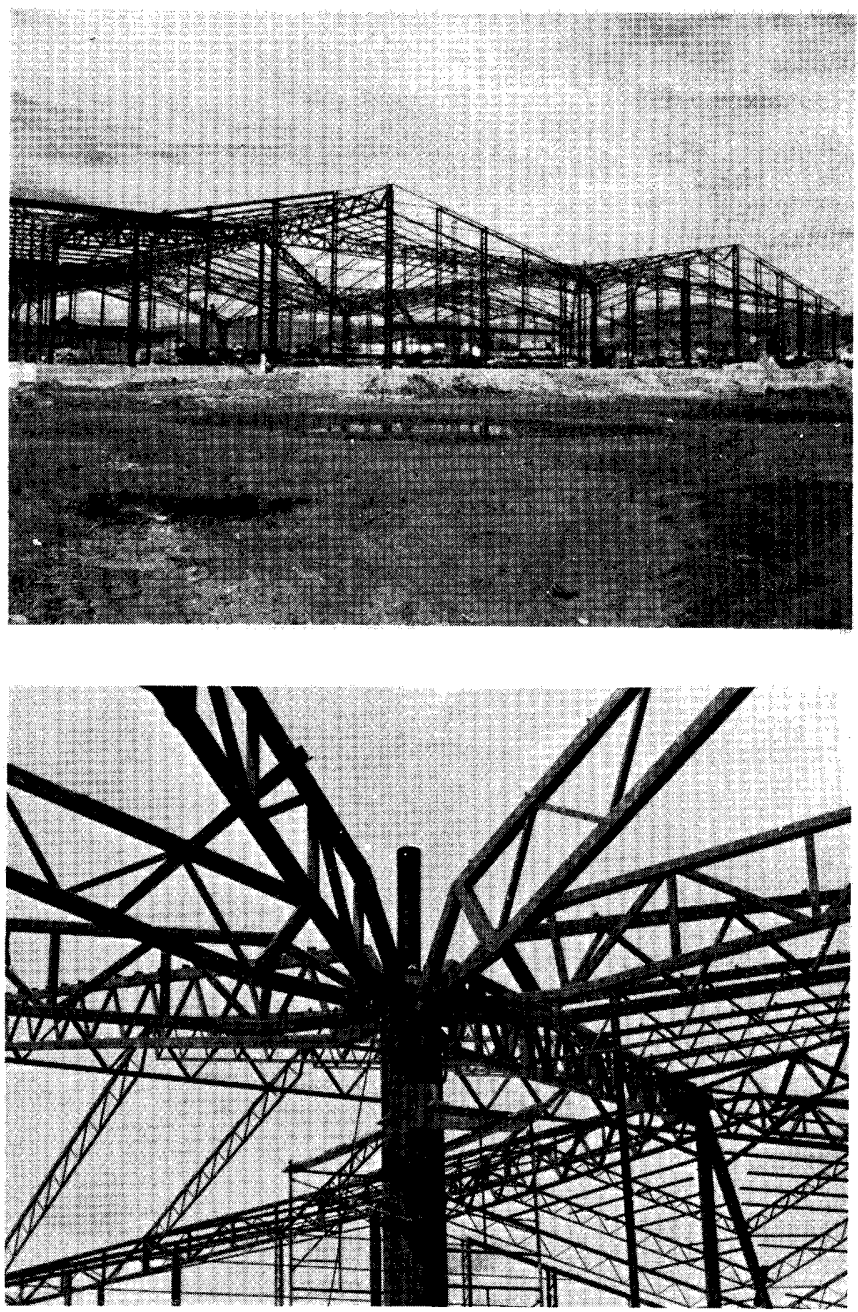

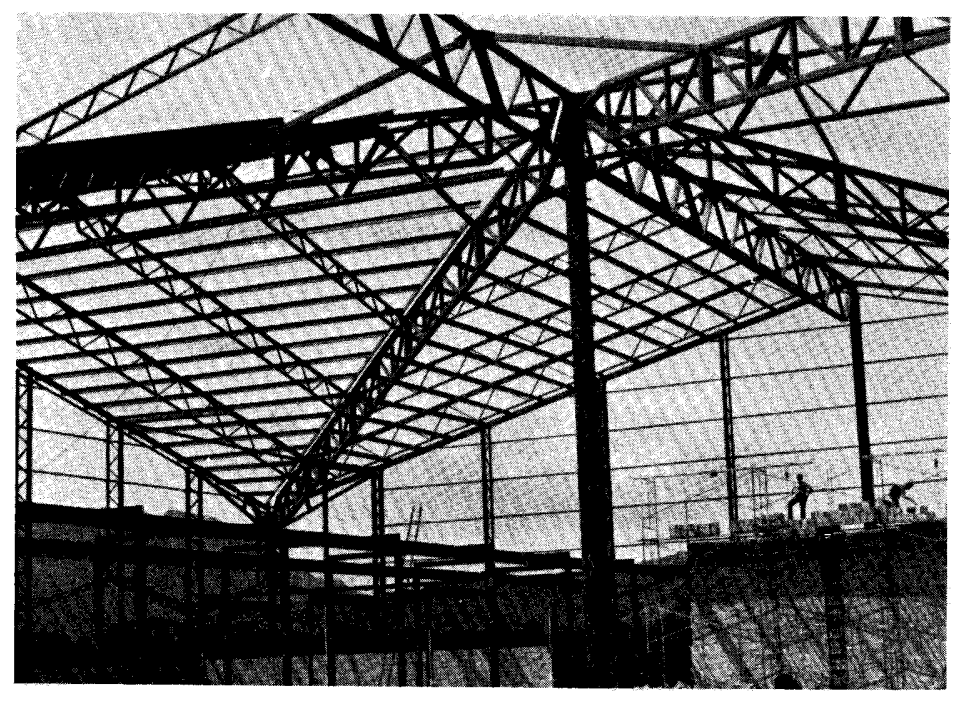

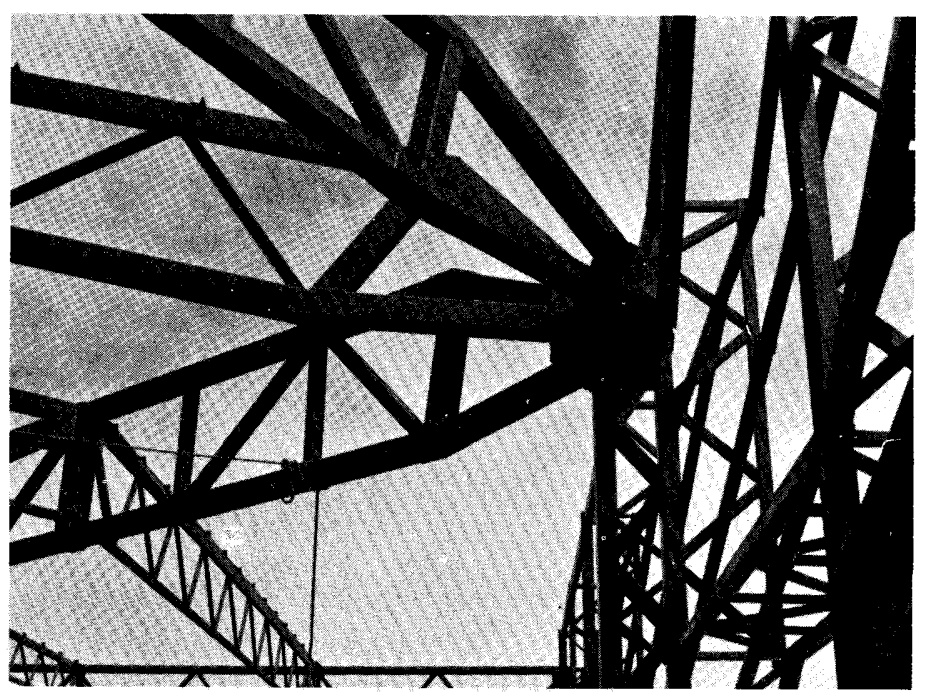



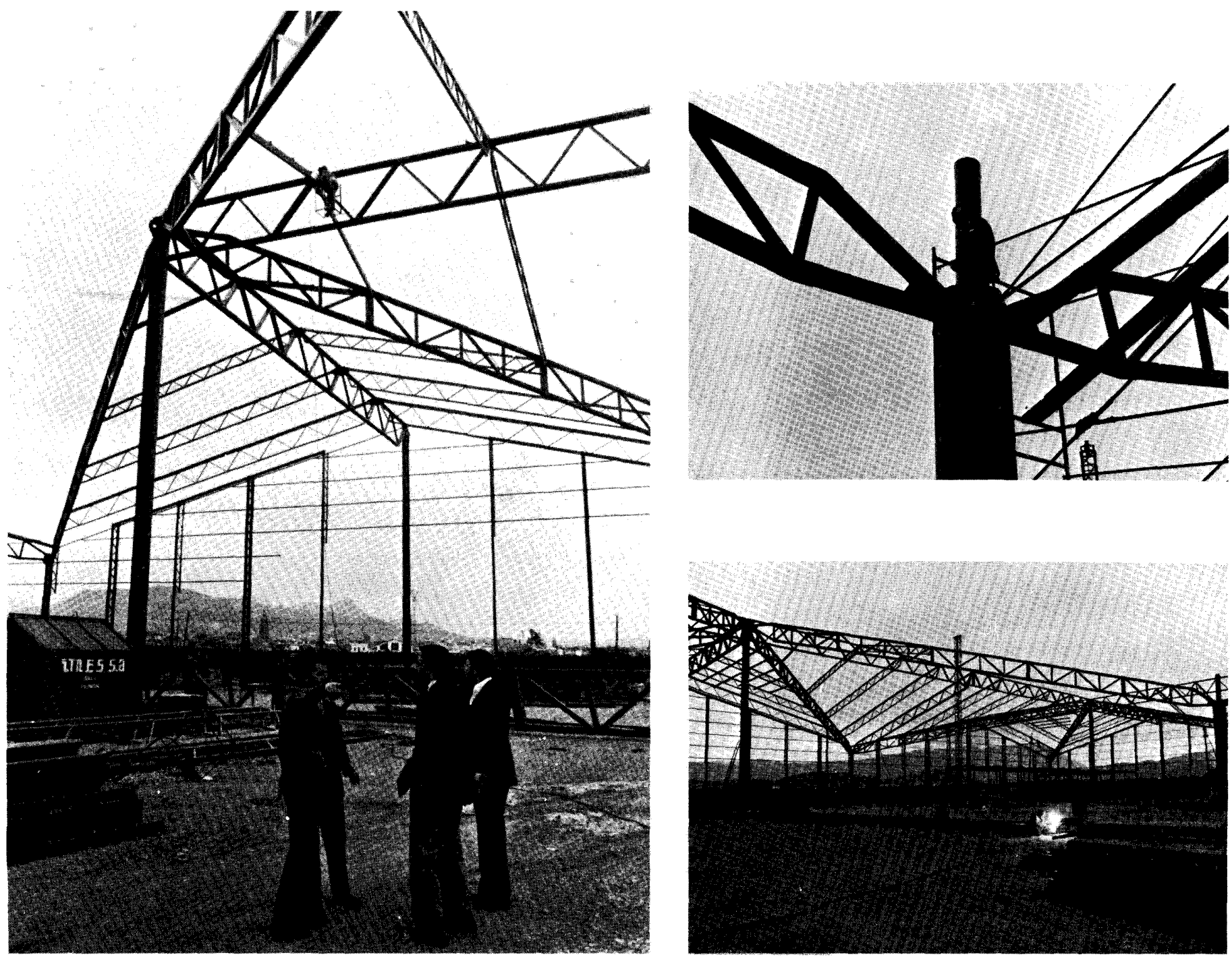

En resumen, en este edificio se conjugan armónicamente funcionalidad técnica y estética que siempre es de desear en las nuevas edificaciones y que raramente se consiguen.

Peso de la estructura del edificio principal $28 \mathrm{~kg} / \mathrm{m}^{2}$, equivalentes a $2,3 \mathrm{~kg} / \mathrm{m}^{3}$.

\section{AGRADECIMIENTOS}

Nuestro agradecimiento al Equipo Humano de ARSEL INGENIERIA en especial al Jefe de Delineación don Carlos Capella, que también ha sabido reflejar en planos la definición de la estructura.

Asimismo queremos destacar el buen hacer de IMES, S. L. constructora de la Estructura Metálica y en especial las muchas horas de dedicación y la ayuda prestada por el Jefe de la Obra don Enrique Rodrigo.

- Promotor y Propietario:

VICENTE Y PASCUAL GINER NAVARRO de Beniarjó (Valencia).

- Constructor Metálico:

IMES, S. L. de Silla (Valencia).

- Ingenieros Proyectistas:

D. CARLOS $M$. FERRER Y FERRER

Dr. Ingeniero Agrónomo.

D. CARLOS A. MARTINEZ LASHERAS

Dr. Ingeniero Agrónomo. 

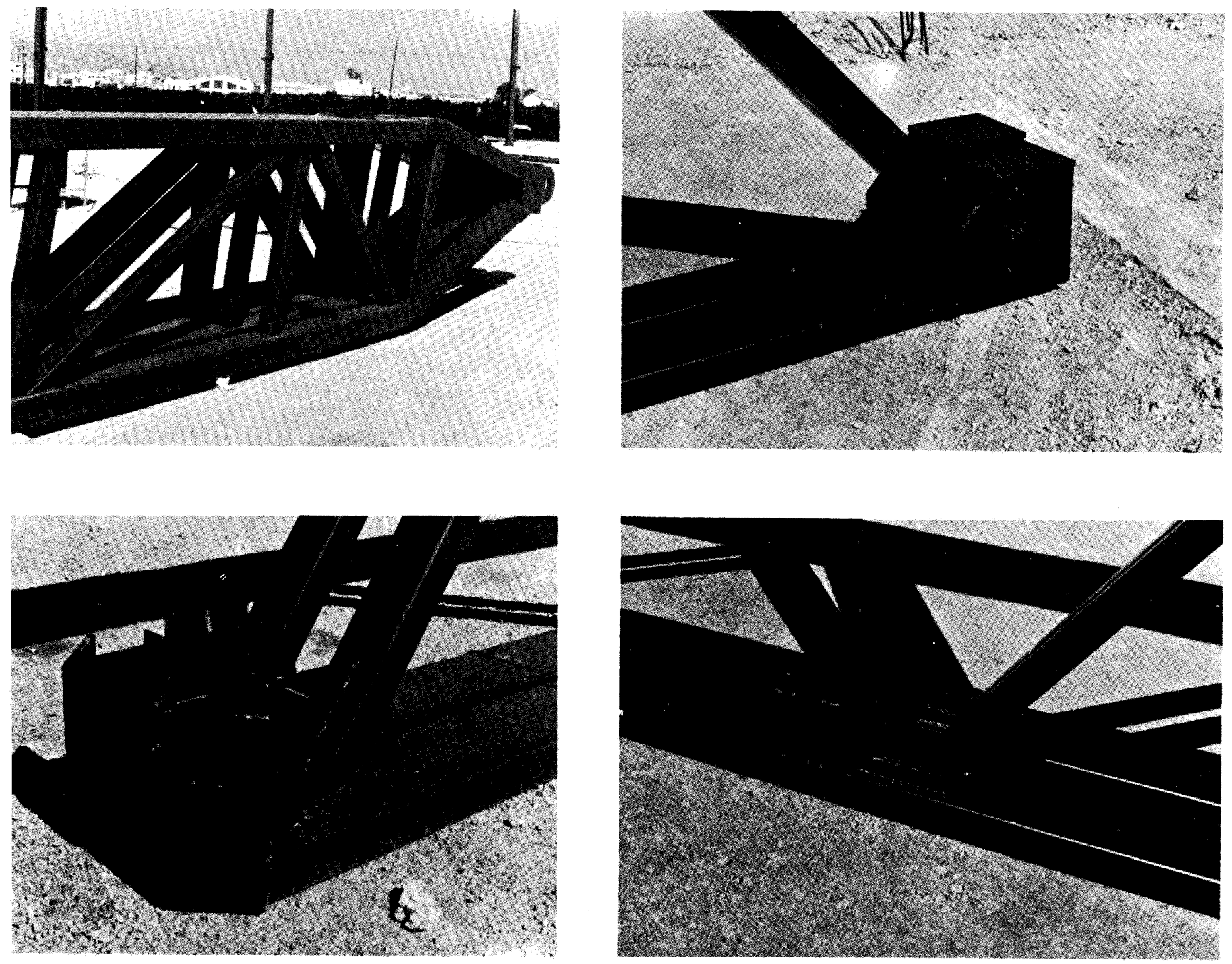

\section{résumé}

OSSATURE METALLIQUE D'UN HALL DESTINE A L'EMMAGÁASINAGE DES FRUITS ET LEGUMES A BENIARJO ESPAGNE

Carlos Ferrer Ferrer et Carlos Martinez Lasheras, Drs. ingénieurs agronomes

La solution originale de ce hall carré qui est ici présentée a remporté le prix Sercometal CECM $/ 80$. Le hall mesure $100 \mathrm{~m}$ de côté et $7.30 \mathrm{~m}$ de hauteur, conçu avec une ossature métallique spatiale constituée par des plans inclinés, résultant ainsi un voile plissé.

Toutes les conditions établies pour sa conception ont été remplies: économie et concurrence avec les ossatures conventionnelles qui laissent les mêmes espaces libres matériau de toiture traditionnel, évacuation correcte des eaux, aspect externe et interne agréables et, en somme, harmonie entre le caractère fonctionnel technique et esthétique, ce qui constitue une reussite exceptionnelle ou au moins non trop fréquente.

\section{summary}

METAL STRUCTURE FOR A FRUIT AND VEGETABLE SHED IN BENIARJO - SPAIN

Carlos Ferrer Ferrer and Carlos Martinez Las heras, Agricultural Engineers

The original design of this shed is presented with a square floor plan, awarded the Sercometal CECM/80 prize, measuring 100 meters on one side and 7.30 meters of useful height, solved on the basis of an overhead metal structure made up by sloped planes, giving the result of a folded sheet.

All of the established premises are fulfilled: economy and competitiveness with conventional structures leaving the same free space, traditional roofing material, proper drainage, pleasant outside and inside appearance and, to sum up, a harmony between the funcional and the aesthetic -an exceptional achievement, or at least one not found too often.

\section{zusammenfassung}

METALLKONSTRUKTION DER CENTRAL HORTOFRUTICOLA IN BENIARJO - SPANIEN

Carlos Ferrer Ferrer und Carlos Martinez Lasheras, Dres. Landwirtschafsingenieure

Es wird hier die originelle Lösung dieser Halle mit quadratischem Grundriss gezeigt, die den Sercometal-CECM/80-Preis erhalten hat. Die Seitenlänge der Konstruktion betragt $100 \mathrm{~m}$ und die Nutzhöhe $7,30 \mathrm{~m}$. Die Lösung besteht in einer räumlichen Metallstruktur, gebildet durch geneigte Ebenen, wodurch eine gefalzte Platte entsteht.

Es sind alle gestellten Voraussetzungen erfüllt; Wirtschaftlichkeit und Konkurrenzfähigkeit mit den herkömmlichen, die dieselben freien Räume belassen, übliches Deckenmaterial, richtiger Wasserablauf, angenehme Gestaltung des Aussen- und Innenausbaus und, kurz gesagt, ein harmonisches Zusammenwirken der funktionellen und ästhetischen Werte, was eine aussergewöhnliche oder zumindest eine nicht allzu häufige Leistung darstellt. 